\title{
Article \\ Is There a Futility Discriminant Function Score for Alcoholic Hepatitis?
}

\author{
Kevin Lamm ${ }^{1}$, Maggie McCarter ${ }^{2}$ and Mark W. Russo ${ }^{1, *}$ \\ 1 Carolinas Medical Center, Department of Internal Medicine, Division of Hepatology, 6th Floor Morehead \\ Medical Plaza, 1025 Morehead Medical Drive, Charlotte, NC 28204, USA; kevin.lamm@atriumhealth.org \\ 2 Carolinas Medical Center, Center for Outcomes Research and Evaluation, Research Office Building, \\ 1000 Blythe Blvd Charlotte, NC 28204, USA; maggie.mccarter@atriumhealth.org \\ * Correspondence: mark.russo@atriumhealth.org; Tel.:+1-704-355-1279; Fax: +1-704-446-4877
}

Citation: Lamm, K.; McCarter, M.; Russo, M.W. Is There a Futility Discriminant Function Score for Alcoholic Hepatitis? J. Clin. Med. 2021, 10, 2756. https://doi.org/ $10.3390 / \mathrm{jcm} 10132756$

Academic Editors: Alan Bonder and Hiroki Nishikawa

Received: 27 April 2021

Accepted: 19 June 2021

Published: 23 June 2021

Publisher's Note: MDPI stays neutral with regard to jurisdictional claims in published maps and institutional affiliations.

Copyright: (c) 2021 by the authors. Licensee MDPI, Basel, Switzerland. This article is an open access article distributed under the terms and conditions of the Creative Commons Attribution (CC BY) license (https:// creativecommons.org/licenses/by/ $4.0 /)$.

\begin{abstract}
The treatment for patients with alcoholic hepatitis $(\mathrm{AH})$ who have a discriminant function (DF) score greater than 32 has been steroids. A prior study reported that mortality approaches $100 \%$ when the DF score is greater than 54, despite the use of prednisolone. Our aim was to determine if a DF score greater than 54 is associated with high mortality despite steroids. We conducted a retrospective study of 531 first-time inpatient encounters of $\mathrm{AH}$. We compared 30-day mortality between patients with DF 54 or less to those greater than 54 treated with steroids, as well as a matched group not treated with steroids. A total of 531 inpatients diagnosed with AH were identified, of which 124 had a DF greater than 32 and 52 were treated with steroids. Among patients treated with steroids, 30-day mortality for patients with DF greater than $54(n=27)$ and 54 or below $(n=25)$ was $22 \%$ and $12 \%$, respectively ( $p=0.47$ ). Among patients with DF greater than 54 , the 30 -day mortality for those who did $(n=27)$ and did not $(n=29)$ receive steroids was $22 \%$ and $24 \%$, respectively $(p=0.87)$. In our study population, steroids were not futile in patients with a DF score of greater than 54 .
\end{abstract}

Keywords: alcoholic hepatitis; discriminant function; futility; mortality

\section{Introduction}

Alcohol use disorder and high-risk drinking rates increased by $36 \%$ and $49 \%$, respectively, over a 10-year span [1]. The National Institute on Alcohol Abuse and Alcoholism analyzed data from 2015 and determined that within the previous month, 56\% of adults reported drinking at least one alcoholic beverage and $27 \%$ admitted to binge drinking [1]. The degree of injury to the liver from alcohol is not linearly dose-dependent, but there appears to be a threshold above which the ongoing consumption of alcohol causes liver injury [2]. For men, the threshold for harmful drinking is four alcohol drinks per day and for women it is two alcohol drinks per day. Binge drinking is defined as men who drink five or more drinks or women who consume four or more drinks in $2 \mathrm{~h}$.

The incidence of alcoholic hepatitis $(\mathrm{AH})$ has been difficult to accurately estimate because its diagnostic coding is not reliable [3]. Pang et al. analyzed a four-and-a-half-year period of hospitalized patients by applying ICD 9 or 10 diagnostic codes and lab criteria. They determined that using ICD codes with laboratory studies was superior to ICD coding alone, with a positive predictive value of $54 \%$. The negative predictive value could not be calculated due to a lack of an unaffected control group [3]. However, the AH consortia validated a protocol using coding and labs that accurately identifies patients with AH [4].

Since Maddrey, et al.'s landmark paper in 1978, patients with a DF score $>32$ have been considered for treatment with corticosteroids [5]. The STOPAH trial evaluated the effect of treatment and found that therapy with prednisolone resulted in a statistically significant decrease in 28-day mortality but did not improve mortality at 90 days or 1 year [6]. A metaanalysis performed by Louvet et al. found that steroids when compared with pentoxifylline 
or placebo showed a decrease in mortality with steroids at 28 days but not at 6 months [7]. Infection has typically been a contraindication to giving steroids for $\mathrm{AH}$ but a meta-analysis by Hmoud et al. suggested a 28-day improvement in mortality in patients with steroids and no difference in mortality based on whether or not patients were treated with steroids [8]. The most recent American Association for the Study of Liver Disease guideline for alcoholic hepatitis does not provide a DF at which treating with steroids is contraindicated [9]. A Veterans Affairs study in 1995 reported one- and six-month mortality based on the DF score, which showed that in the range of 35-54, mortality was reduced with steroids, but those with a DF score $>54$ steroids did not obtain benefits and these scores were associated with lower survival, suggesting a threshold above which corticosteroids are harmful [10]. Twenty-five years have elapsed since this study and significant advances have been made in treating advanced liver disease. The aim of this study was to determine if the use of steroids was futile in patients with an $\mathrm{AH}$ and DF greater than 54 .

\section{Patients and Methods}

\subsection{Patient Selection}

We identified AH patients who were hospitalized during January 2010-December 2018, using the Atrium Health Electronic Medical Record. The Atrium Health Electronic Medical Record includes information from all hospitals within the healthcare system including Carolinas Medical Center and 11 regional hospitals, with a combined total of 3000 beds. We excluded patients less than age 18 .

\subsection{Definition of $A H$}

To determine the diagnosis of $\mathrm{AH}$, we applied the AH consortia methodology [4]. We screened patients with the ICD 9 and ICD 10 codes (571.1 and K70.1), including those that that had obtained a primary or secondary diagnosis from Atrium Health's Electronic Medical Record. A total of 531 patients were identified. To increase the study's specificity, only patients with the following lab values were included in the study: AST $>50 \mathrm{U} / \mathrm{L}$, AST $<400 \mathrm{U} / \mathrm{L}, \mathrm{ALT}<400 \mathrm{U} / \mathrm{L}, \mathrm{T}$. Bili $>3 \mathrm{mg} / \mathrm{dL}$, and a AST $/$ ALT ratio of $>1.5$, as recommended by the AH consortia [4]. Afterwards, 260 records were utilized after screening with the inclusion criteria (Figure 1).

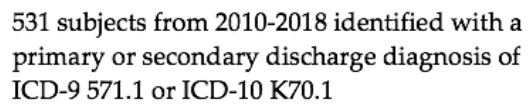

260 subjects with alcoholic hepatitis identified

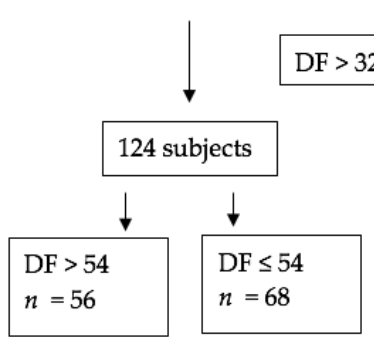

Figure 1. Study flow diagram. 


\subsection{Data Collection}

Data was collected on patient demographics as well as 30- and 90-day mortality. Laboratory values analyzed included white blood cell count, platelets, sodium, creatinine, prothrombin time, INR, bilirubin day 0 and 7, albumin, ALT, and AST. The prognostic scores were calculated for all patients using the first lab taken after inpatient admission. Afterwards, 30- and 90-day mortality were gathered by using the social security database. Presence of infection or GI hemorrhage was defined by ICD 9 and ICD 10 codes (see Appendix A). The Charlson Comorbidity Index was calculated using the Atrium Healthcare ICD 9 and 10 coding algorithms. All data entry and storage were kept secure in an Excel spreadsheet.

\subsection{Objective of the Study}

The primary endpoint of the study was 30-day mortality. The secondary endpoint was 90-day mortality. The objective of the study was to determine if there is a DF score at which giving steroids is futile.

\subsection{Statistical Analysis}

Using descriptive statistics and univariate analyses, baseline characteristics were compared between the 33-54 and $>54$ DF score groups in patients who received steroids, and between steroid- and no-steroid treatment groups in patients with a DF greater than 54 Continuous variables are reported as medians and interquartile ranges, and categorical variables are reported as frequencies and percentages. Between-group comparisons for baseline characteristics were assessed using Student's $t$-test for normally distributed variables, non-parametric Kruskal Wallis tests for continuous variables that were not normally distributed, and Pearson's chi-square test or Fisher's exact tests for categorical variables. The groups were compared on 30-day and 90-day mortality using the chi-square test or Fisher's exact test. Primary analyses of the relationship between DF, Creatinine, Albumin, and steroid treatment, and 30- and 90-day mortality among those with a DF $>32$ were performed using multiple logistic regression models adjusting for GI bleeding, age, and the Charlson Comorbidity Index. Secondary analyses of the relationship between DF and 30- and 90-day mortality among those treated with steroids were performed using multiple logistic regression models adjusting for age and creatinine lab value, and the Charlson Comorbidity Index and creatinine separately. All statistical tests were two-sided with a significance level of 0.05, and were conducted using SAS/STAT, Version 9.4 (SAS Institute Inc., Cary, NC, USA).

\section{Results}

\subsection{Study Population}

From January 2010 through December 2018, 531 patients were given a primary or secondary discharge diagnosis of $\mathrm{AH}$. Of those, 260 met the laboratory inclusion criteria. In addition, 124 of those patients had DF > 32 with 56 and 68 patients had DF > 54 and DF 54 or less, respectively. Baseline characteristics of the 52 patients who received steroids with DF greater than 32 are shown in Table 1, stratified by a DF score of 33-54 and greater than 54 . Variables that were significantly different between groups include serum sodium, creatinine, PT/INR, albumin, and diagnosis of infection (defined in Appendix A). Baseline characteristics of patients with DF greater than 54 are summarized in Table 2 and compared based on whether or not they received steroids. Patients who received steroids had a significantly lower serum sodium, higher serum AST, and a higher percentage of diagnosed infection. 
Table 1. Baseline Characteristics of Patients Who Received Steroids with DF $>32$.

\begin{tabular}{|c|c|c|c|c|}
\hline & $\begin{array}{c}\text { Total } \\
n=52\end{array}$ & $\begin{array}{c}\text { DF } 33-54 \\
n=25\end{array}$ & $\begin{array}{c}\text { DF } 55+ \\
n=27\end{array}$ & $p$-Value \\
\hline Age, years (IQR) & $47(36,52)$ & $47(37,52)$ & $45(34,51)$ & 0.36 \\
\hline Female $(\%)$ & $15(29)$ & $7(28)$ & $8(30)$ & 0.90 \\
\hline \multicolumn{5}{|l|}{ Race } \\
\hline Black (\%) & $8(15)$ & $4(16)$ & $4(15)$ & 0.85 \\
\hline White (\%) & $43(83)$ & $20(80)$ & $23(85)$ & \\
\hline \multicolumn{5}{|l|}{ Ethnicity } \\
\hline Declined (\%) & $3(6)$ & $1(4)$ & $2(7)$ & 0.062 \\
\hline Hispanic or Latino (\%) & $4(8)$ & $4(16)$ & $0(0)$ & \\
\hline Non-Hispanic or Latino (\%) & $45(87)$ & $20(80)$ & $25(93)$ & \\
\hline WBC Count $10^{3} / \mu \mathrm{L}$ (IQR) & $9.8(7.5,14.9)$ & $9.2(6.1,14.7)$ & $10.6(8.1,15.2)$ & 0.28 \\
\hline Platelets $10^{3} / \mu \mathrm{L}$ (IQR) & $127(69,182)$ & $133(98,206)$ & $123(65,157)$ & 0.14 \\
\hline Sodium mmol/L (IQR) & $130(128,134)$ & $131(129,135)$ & $129(125,132)$ & 0.02 \\
\hline Creatinine mg/dL (IQR) & $1.0(1.0,1.3)$ & $1.0(1.0,1.0)$ & $1.0(1.0,1.9)$ & 0.01 \\
\hline Prothrombin Time seconds (IQR) & $21.0(19.1,22.4)$ & $19.2(17.7,20.0)$ & $22.2(21.7,25.0)$ & $<0.001$ \\
\hline INR & $1.9(1.7,2.1)$ & $1.6(1.5,1.7)$ & $2.0(1.9,2.2)$ & $<0.001$ \\
\hline Bilirubin Day 0 mg/dL (IQR) & $20.8(14.1,25.5)$ & $19.9(11.0,21.8)$ & $22.2(15.8,27.7)$ & 0.06 \\
\hline Lille Score & $0.69(0.26,0.96)$ & $0.42(0.23,0.79)$ & $0.95(0.55,0.99)$ & 0.11 \\
\hline AST & $164(117,239)$ & $136(91,213)$ & $171(137,274)$ & 0.14 \\
\hline Albumin g/dL (IQR) & $2.2(1.9,2.5)$ & $2.3(2.1,2.5)$ & $2.0(1.8,2.4)$ & 0.045 \\
\hline Presence of GI Hemorrhage (\%) & $5(10)$ & $3(12)$ & $2(7)$ & 0.66 \\
\hline Infection & $13(25)$ & $1(4)$ & $12(44)$ & $<0.001$ \\
\hline \multicolumn{5}{|l|}{ Median MELD } \\
\hline $20-29(\%)$ & $41(79)$ & $24(96)$ & $17(63)$ & $<0.001$ \\
\hline $30+(\%)$ & $10(19)$ & $0(0)$ & $10(37)$ & \\
\hline Median MELD-Na & & & & $<0.001$ \\
\hline $20-29(\%)$ & $36(69)$ & 25 (100) & $11(41)$ & $<0.001$ \\
\hline $30+(\%)$ & $16(31)$ & $0(0)$ & $16(59)$ & \\
\hline
\end{tabular}

Table 2. Baseline Characteristics of Patients with DF > 54 .

\begin{tabular}{|c|c|c|c|c|}
\hline & $\begin{array}{c}\text { Total } \\
n=56\end{array}$ & $\begin{array}{c}\text { No Steroids } \\
n=29\end{array}$ & $\begin{array}{c}\text { Steroids } \\
n=27\end{array}$ & $p$-Value \\
\hline Age, years (IQR) & $46(37,51)$ & $46(40,50)$ & $45(34,51)$ & 0.27 \\
\hline Female (\%) & $19(34)$ & $11(38)$ & $8(30)$ & 0.51 \\
\hline Race & & & & 0.67 \\
\hline Black (\%) & $6(11)$ & $2(7)$ & $4(15)$ & 0.67 \\
\hline White (\%) & $48(89)$ & $25(93)$ & $23(85)$ & \\
\hline \multicolumn{5}{|l|}{ Ethnicity } \\
\hline Declined (\%) & $5(9)$ & $3(10)$ & $2(7)$ & 0.67 \\
\hline Hispanic or Latino (\%) & $2(4)$ & $2(7)$ & $0(0)$ & \\
\hline Non-Hispanic or Latino (\%) & $49(87)$ & $24(83)$ & $25(93)$ & \\
\hline WBC Count $10^{3} / \mu \mathrm{L}$ (IQR) & $10.6(7.5,14.8)$ & $10.6(7.3,13.5)$ & $10.6(8.1,15.2)$ & 0.79 \\
\hline Platelets $10^{3} / \mu \mathrm{L}$ (IQR) & $125(79,157)$ & $128(79,162)$ & $123(65,157)$ & 0.36 \\
\hline Sodium mmol/L (IQR) & $131(128,134)$ & $132(130,134)$ & $129(125,132)$ & 0.001 \\
\hline Creatinine mg/dL (IQR) & $1.0(1.0,2.0)$ & $1.0(1.0,2.1)$ & $1.0(1.0,1.9)$ & 0.32 \\
\hline Prothrombin Time seconds (IQR) & $23.8(21.6,27.3)$ & $25.5(21.4,27.7)$ & $22.2(21.7,25.0)$ & 0.28 \\
\hline INR & $2.2(1.9,2.6)$ & $2.4(2.1,2.6)$ & $2.0(1.9,2.2)$ & 0.18 \\
\hline Bilirubin Day 0 mg/dL (IQR) & $22.2(14.9,27.6)$ & $21.9(14.0,27.5)$ & $22.2(15.8,27.7)$ & 0.43 \\
\hline AST & $166(123,209)$ & $145(111,199)$ & $171(137,274)$ & 0.03 \\
\hline Albumin g/dL (IQR) & $2.0(1.8,2.3)$ & $2.0(1.8,2.3)$ & $2.0(1.8,2.4)$ & 0.97 \\
\hline Presence of GI Hemorrhage (\%) & $3(5)$ & $1(3)$ & $2(7)$ & 0.60 \\
\hline Infection & $15(27)$ & $3(10)$ & $12(44)$ & 0.004 \\
\hline \multicolumn{5}{|l|}{ Median MELD } \\
\hline $20-29(\%)$ & $35(62)$ & $18(62)$ & $17(63)$ & 0.94 \\
\hline $30+(\%)$ & $21(38)$ & $11(38)$ & $10(37)$ & \\
\hline \multicolumn{5}{|l|}{ Median MELD-Na } \\
\hline $20-29(\%)$ & $25(45)$ & $14(48)$ & $11(41)$ & 0.57 \\
\hline $30+(\%)$ & $31(55)$ & $15(52)$ & $16(59)$ & \\
\hline
\end{tabular}

\subsection{Patient Survival}

Figure 2 compares 30-day and 90-day mortality based on the DF score $(\leq 54,>54)$ and steroid treatment (yes/no). For patients with DF $>54$ who did $(n=27)$ and did not $(n=29)$ receive steroids, 30 -day mortality was $22 \%$ and $24 \%$, respectively ( $p=0.87$ ). For those with 
$\mathrm{DF} \leq 54$ but $>32,30$-day mortality for those who did $(n=25)$ and did not $(n=43)$ receive steroids was $12 \%$ and $16 \%$, respectively $(p=0.66)$.

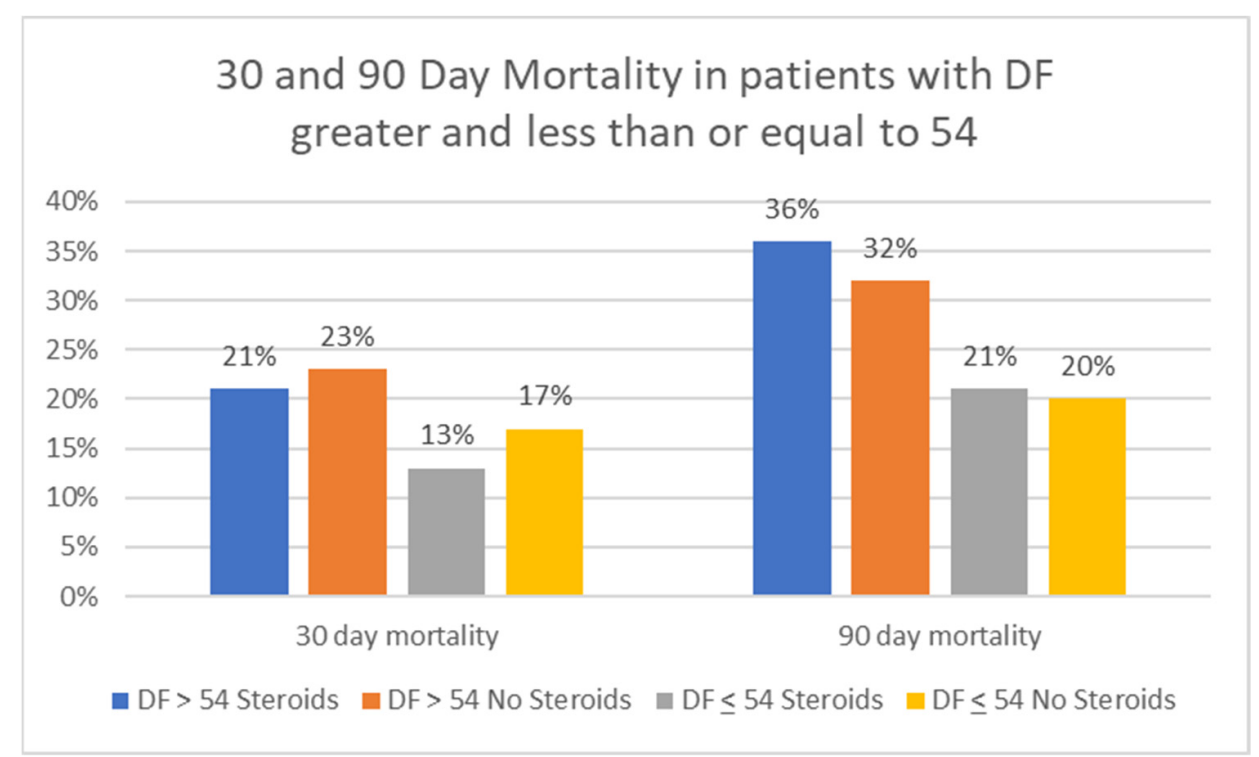

Figure 2. 30- and 90-day mortality comparing those who did and did not receive steroids as well as DF $>$ and $<54$.

In an additional effort to find a DF score that would suggest futility, we compared outcomes in patients with a DF score $>65$. For patients with DF $>65$ who did $(n=15)$ and did not $(n=17)$ receive steroids, 30-day mortality was $20 \%$ and $29 \%$, respectively $(p=0.69)$. Table 3 shows results of the multivariable logistic regression analyses for $30-$ and 90-day mortality in patients with DF $>32$. Creatinine on admission was found to have a statistically significant impact on 30-day mortality, but not on 90-day mortality. Based on the International Club of Ascites defining acute kidney injury as an absolute increase in serum $\mathrm{Cr}$ of $0.3 \mathrm{mg} / \mathrm{dL}$ or more from baseline, we chose to look at whether an increase of $0.3 \mathrm{mg} / \mathrm{dL}$ of creatinine led to increased mortality [11]. For every 0.3-unit increase in creatinine, the odds of 30-day mortality increased by $19 \%$ (OR 1.19 95\%CI 1.04-1.37). No other statistically significant associations were found.

Table 3. Logistic Regression of 30- and 90- day mortality in patients with DF > 32 .

\begin{tabular}{ccc}
\hline \multicolumn{2}{c}{ Logistic Regression of 30-Day Mortality among Patients with DF > 32 } & 95\% CI $(p$-Value $)$ \\
\hline Variable & Odds Ratio & $0.65-4.32(0.29)$ \\
\hline DF $>$ 54 vs. DF 32-54 & 1.67 & $1.04-1.37(0.01)$ \\
\hline Creatinine (0.3 unit increase) & 1.19 & $0.37-3.70(0.80)$ \\
\hline Albumin & 1.16 & $0.39-2.61(0.98)$ \\
\hline Steroid Treatment & 1.01 & $\mathbf{9 5 \%}$ CI ( $p$-Value) \\
\hline \multicolumn{1}{c}{ Logistic Regression of 90-Day Mortality among Patients with DF > 32 } \\
\hline Variable & Odds Ratio & $0.88-4.79(0.10)$ \\
\hline DF $>$ 54 vs. DF 32-54 & 2.05 & $1.00-1.28(0.06)$ \\
\hline Creatinine (0.3 unit increase) & 1.13 & $0.42-3.20(0.78)$ \\
\hline Albumin & 1.15 & $0.64-3.33(0.37)$
\end{tabular}


Results of the logistic regressions performed on the relationship between 30- and 90-day mortality and DF within patients treated with steroids can be found in Table 4 . There were no significant associations between DF and 30- and 90-day mortality.

Table 4. Logistic Regression of the Relationships between 30- and 90-Day Mortality and DF in Patients Treated with Steroids.

\begin{tabular}{ccc}
\hline Outcome & Odds Ratio & $\mathbf{9 5 \% ~ C I ~ ( ~} \boldsymbol{p}$-Value) \\
\hline 30-Day Mortality * & 0.98 & $0.9-1.03(0.42)$ \\
\hline 90-Day Mortality * & 0.98 & $0.94-1.02(0.33)$ \\
\hline 30-Day Mortality ** & 0.98 & $0.94-1.03(0.40)$ \\
\hline 90-Day Mortality ** & 0.98 & $0.94-1.02(0.23)$ \\
\hline
\end{tabular}

* Controlling for age and creatinine. ${ }^{* *}$ Controlling for CCI and creatinine.

\section{Discussion}

Alcoholic hepatitis is a common consult for gastroenterologists and for more than four decades, the decision of whether to initiate steroids has been a large part of its management. The controversy over steroids persists and whether steroids offer a survival benefit or do more harm than good continues to be discussed. Studies have demonstrated short term survival, or 30-day survival can be improved, but may come at the expense of increased infections, and long-term survival as 90-day or 6-month survival is not improved with steroids. Whether there is a point of futility or a DF score where severity of AH is so severe that steroids will not improve outcomes is not well described.

The DF score is applied as a dichotomous variable whereby individuals with a DF score greater than 32 are considered for steroids and those with scores 32 or less receive supportive therapy alone. However, a patient with a DF score of 55 may be much sicker than one with a score of 35. In a VA study that included 536 patients with $\mathrm{AH}$ and compared placebo, prednisolone, and oxandrolone, mortality was significantly lower in patients treated with prednisolone with DF between 35 and 54 compared to the placebo group. In patients with DF greater than 54, mortality was higher in the prednisolone group and approached $100 \%$ compared to a mortality of $75 \%$ in the placebo group, but it was not reported to be statistically significant [10]. In our study, in patients with DF $>54$ mortality was $21 \%$ and $23 \%$ in patients treated with steroids and those not treated with steroids. Although our study suggests steroids do not improve survival in patients with DF $>54$, larger prospective studies are needed to confirm this finding before definitive recommendations could be made to withhold steroids from patients with $\mathrm{AH}$ and DF scores $>54$.

Since the development of the DF score, a number of other prognostic scores have been developed, but none have replaced the DF. A Glasgow Alcoholic Hepatitis Score of 9 or higher or a MELD score of 20 or higher have been proposed to select patients for steroids or liver-specific treatment for $\mathrm{AH}$, but neither are substantially better at predicting mortality compared to the DF score $[12,13]$. The Lille Score at baseline and day 4 or 7 allows the clinician to assess the response to steroids. Non-responders can stop steroids and alternative therapies such as early liver transplantation should be considered [14]. For the most part, the DF score has stood the test of time and remains in guidelines for identifying patients with AH who may benefit from steroids [9].

The original study that developed DF reported improved survival in patients with AH with scores greater than 32. Patients were excluded if they had active gastrointestinal bleeding, pancreatitis, a history of peptic ulcer disease, active infection, or a history of viral hepatitis. The 55 subjects included in the study were divided into three groups based on severity of illness defined by presence of ascites, encephalopathy, coagulopathy, and bilirubin. Higher mortality was associated with encephalopathy, coagulopathy, hypoalbuminemia, higher bilirubin, higher BUN, and creatinine. All deaths occurred in patients 
with a DF score of 93 or higher; 6 of 8 placebo patients died and 1 or 7 prednisolone patients died $(p=0.03)$ [5].

The limitations of our study include those associated with retrospective studies and the biases associated with selecting a control group. Patients with DF $>54$ who were selected to receive steroids may have been systematically different than patients with DF $>54$ who did not receive steroids. Various confounders were controlled for to address this bias. Creatinine is not included in the DF score, but it is included in the MELD score and the effect of renal injury on futility of steroids in AH is worth further study with MELD. Cases were identified using ICD codes; however, we used rigorous methods previously identified by the Alcohol Research Consortium that have been validated and have excellent accuracy for identifying patients with $\mathrm{AH}[3,4]$. Although our study included a large number of overall patients with $\mathrm{AH}$, the number of patients in subgroups was smaller and we may have been underpowered to detect a clinically significant difference between groups with high and low DF scores.

In conclusion, in contrast to the VA study, we did not find that patients with $\mathrm{AH}$ and DF scores greater than 54 had higher mortality compared to those with lower DF scores. Even in patients with severe $\mathrm{AH}$, steroids do not appear to be futile. We advocate all candidates without contraindications be considered for a trial of steroids and evaluated for responsiveness with a 4- or 7-day Lille Score. Continued development and refinement of prognostic scores that can identify patients at greatest risk of death despite steroids or other future therapies need to be developed to identify patients that would obtain the greatest benefit or those where therapy is futile.

Author Contributions: Conceptualization, M.W.R.; methodology, K.L.; software, M.M.; validation, M.W.R., K.L. and M.M.; formal analysis, M.W.R. and M.M.; investigation, K.L. and M.W.R.; data curation, K.L. and M.M.; writing—original draft preparation, K.L. and M.W.R.; writing-review and editing, M.W.R.; supervision, M.W.R.; project administration, M.W.R. All authors have read and agreed to the published version of the manuscript.

Funding: This research received no external funding.

Institutional Review Board Statement: The study was conducted according to the guidelines of the Declaration of Helsinki, and approved by the Institutional Review Board of Atrium Health IRB File \#: 09-17-14E approved on 21 July 2018.

Informed Consent Statement: Patient consent was waived because the signature on the informed consent document would be the only record linking the subject to the research and the principal risk of harm to the subject would be a breach of confidentiality.

Data Availability Statement: The data presented in this study are available on request from the corresponding author. The data are not publicly available due to privacy.

Conflicts of Interest: The authors declare no conflict of interest.

\section{Appendix A}

ICD Codes used for the presence of GI Bleeding: K92.0; K92.2; I85.01; K29.01; K29.21; K29.51; K29.81; K25.0; K25.2 K25.4; K26.4; K22.6; K22.11; 578.0; 578.9; 456.0; 535.01; 535.31; $535.11 ; 535.61 ; 531.01 ; 531.20 ; 531.40 ; 532.40 ; 530.7 ; 530.21$

ICD Codes used for the presence of infection: K65.2; J18.9; J15; A41.9; R78.81; 567.23; 482; 995.91; 038.9; 790.7

\section{References}

1. Grant, B.F.; Chou, S.P.; Saha, T.D.; Pickering, R.P.; Kerridge, B.T.; Ruan, W.J.; Hasin, D.S. Prevalence of 12-Month Alcohol Use, High-Risk Drinking, and DSM-IV Alcohol Use Disorder in the United States, 2001-2002 to 2012-2013: Results from the National Epidemiologic Survey on Alcohol and Related Conditions. JAMA Psychiatry 2017, 74, 911-923. [CrossRef] [PubMed]

2. Rehm, J.; Taylor, B.; Mohapatra, S.; Irving, H.; Baliunas, D.; Patra, J.; Roerecke, M. Alcohol as a risk factor for liver cirrhosis: A systematic review and meta-analysis. Drug Alcohol Rev. 2010, 29, 437-445. [CrossRef] [PubMed] 
3. Pang, J.X.; Ross, E.; Borman, M.A.; Zimmer, S.; Kaplan, G.G.; Heitman, S.J.; Myers, R.P. Validation of coding algorithms for the identification of patients hospitalized for alcoholic hepatitis using administrative data. BMC Gastroenterol. 2015, 15, 116-120. [CrossRef] [PubMed]

4. Crabb, D.W.; Bataller, R.; Chalasani, N.P.; Kamath, P.S.; Lucey, M.; Mathurin, P.; Szabo, G. Standard definitions and common data elements for clinical trials in patients with alcoholic hepatitis: Recommendation from the NIAAA alcoholic hepatitis consortia. Gastroenterology 2016, 150, 787-790. [CrossRef] [PubMed]

5. Maddrey, W.C.; Boitnott, J.K. Corticosteroid therapy of alcoholic hepatitis. Gastroenterology 1978, 75, 193-199. [CrossRef]

6. Thursz, M.R.; Richardson, P.; Allison, M.; Austin, A.; Bowers, M.; Day, C.P.; Downs, N.; Gleeson, D.; MacGilchrist, A.; Grant, A.; et al. Prednisolone or pentoxifylline for alcoholic hepatitis. N. Engl. J. Med. 2015, 372, 1619-1628. [CrossRef] [PubMed]

7. Louvet, A.; Thursz, M.R.; Kim, D.J.; Labreuche, J.; Atkinson, S.R.; Sidhu, S.S.; Mathurin, P. Corticosteroids Reduce Risk of Death Within 28 Days for Patients with Severe Alcoholic Hepatitis, Compared with Pentoxifylline or Placebo-a Meta-analysis of Individual Data from Controlled Trials. Gastroenterology 2018, 155, 458-468. [CrossRef] [PubMed]

8. Hmoud, B.S.; Patel, K.; Bataller, R.; Singal, A.K. Corticosteroids and occurrence of and mortality from infections in severe alcoholic hepatitis: A meta-analysis of randomized trials. Liver Int. 2016, 36, 721-728. [CrossRef]

9. Crabb, D.W.; Im, G.Y.; Szabo, G.; Mellinger, J.L.; Lucey, M.R. Diagnosis and Treatment of Alcohol-Associated Liver Diseases: 2019 Practice Guidance from the American Association for the Study of Liver Diseases. Hepatology 2020, 71, 306-333. [CrossRef] [PubMed]

10. Mendenhall, C.; Roselle, G.A.; Gartside, P.; Moritz, T. Relationship of protein calorie malnutrition to alcoholic liver disease: A reexamination of data from two Veterans Administration Cooperative Studies. Alcohol. Clin. Exp. Res. 1995, 19, 635-641. [CrossRef] [PubMed]

11. Angeli, P.; Ginès, P.; Wong, F.; Bernardi, M.; Boyer, T.D.; Gerbes, A.; Garcia-Tsao, G. Diagnosis and management of acute kidney injury in patients with cirrhosis: Revised consensus recommendations of the International Club of Ascites. J. Hepatol. 2015, 62, 968-974. [CrossRef] [PubMed]

12. Forrest, E.G.; Morris, A.J.; Stewart, S.; Phillips, M.; Oo, Y.H.; Fisher, N.C.; Day, C.P. The Glasgow alcoholic hepatitis score identifies patients who may benefit from corticosteroids. Gut 2007, 56, 353-358. [CrossRef] [PubMed]

13. Dunn, W.; Jamil, L.H.; Brown, L.S.; Wiesner, R.H.; KIM, W.R.; Menon, K.V.; Shah, V. MELD accurately predicts mortality in patients with alcoholic hepatitis. Hepatology 2005, 41, 353-358. [CrossRef] [PubMed]

14. Louvet, A.; Naveau, S.; Abdelnour, M.; Ramound, M.J.; Diaz, E.; Fartoux, L.; Mathurin, P. The Lille model: A new tool for therapeutic strategy in patients with severe alcoholic hepatitis treated with steroids. Hepatology 2007, 45, 1348-1354. [CrossRef] [PubMed] 